werden! Bei der Abwasserbeseitigung ist man inzwischen dazu übergegangen, anfallendes Methan zu gewinnen. Denken Sie aber auch an die Müllverarbeitung; den Anfall organischer Produkte, die man auch in irgendeiner Weise weiterverwenden kann, insbesondere als Nährstoffe für Bakterien etc., oder denken Sie an die Massenzüchtung von Algen, wie sie z. B. im Institut für Kohlenstofforschung betrieben wird! Dies sind Fragen, die in Zukunft eine große Rolle spielen werden. Ich glaube, wir werden es in den nächsten Jahren jedenfalls nicht erleben, daß wir arbeitslos werden.

\title{
Methodik der Bearbeitung sulfatreduzierender Bakterien und mikrobielle Aktivitäten in Gasspeichern
}

GunKeL: Welche Kohlenstoffquellen verwenden Sie in Ihren 'Testbestecken, Herr WaLLeÄUsSER?

Wallhäusser: Nur Calciumlactat. Herr Professor BaArs äußerte übrigens, daß Calciumacetat auch sehr gut geeignet sei. Wir werden das prüfen. Manche Autoren sind der Meinung, Methan werde durch diese Organismen ausgenutzt. Wir wissen noch nicht, welcher Bestandteil vom Speichergas oder vom Erdöl als beste Kohlenstoffquelle dient. Es ist etwas enttäuschend zu sehen, wie wenig bei einem Abbauversuch im Boden geschieht. Andererseits kennen wir die ausgezeichnete Entwicklung dieser Desulfovibrionen in den Untertagegasspeichern, wo dex durch sie angerichtete Schaden erheblich ist. In einem Jahr fallen in einem Untertagegasspeicher mit 200 Millionen Norm $\mathrm{m}^{3}$ etwa 20 Tonnen Schwefelwasserstoff an. In Lacq (Südfrankreich) gibt es ein natürliches Erdgasvorkommen, das bis zu $15 \% \mathrm{H}_{2} \mathrm{~S}$ hat. Dort wird $\mathrm{H}_{2} \mathrm{~S}$ schon regelrecht gefördert. Man gewinnt daraus Schwefel, und ich habe ausgerechnet, daß ungefähr 16000 Jahre notwendig sind, um einen Schwefelgehalt von $15 \%$ zu erzielen. Früher hatte man sich vom Gesamtgeschehen ganz andere Vorstellungen gemacht; man hatte an eine Lagerung uber Jahrmillionen gedacht, bis ein solch hoher Schwefelwasserstoffgehalt zuwege gebracht ist. Weit gefehlt! Wir haben gerade durch diese Erfahrungen in den Untertagegasspeichern gesehen, $\mathrm{d} a ß$ dieses Geschehen zuweilen viel schneller abläuft, als man es wünscht, und zwar meist in negativer, manchmal aber auch in positiver Hinsicht. Denn auf der anderen Seite schlägt die Industrie ihr Kapital daraus, wenn sie beispielsweise Antibiotika oder Vitamine herstellt.

GUNKeL: Unter welchem Druck stehen die Bakterien in unterirdischen Gasspeichern bei 120 bis $140^{\circ} \mathrm{C}$ ?

WALLhÄUSSER: Der Druck beträgt etwa 100 bis $120 \mathrm{~atm}$.

GUNKeL: Sie kennen sicher die Arbeiten von ZoBeLL, der bei stark erhöhtem Druck eine erhöhte Resistenz gegen Hitze fand.

WallhäUsSER: Ich darf Thnen vorlesen, was ich dazu geschrieben habe: „Zur Erklärung seien einige amerikanische Untersuchungen angeführt. So konnte ZoBeli zeigen, daß diese Organismen hohe Temperaturen bei hohem Druck weit besser überstehen als beispielsweise bei $1 \mathrm{~atm}$." Wir Mikrobiologen sind immer etwas skeptisch gegenüber solch hohen Temperaturen und Drücken zugleich, denn wir wissen vom Autoklavieren: je höher der Druck wird, desto kürzer die Abtötungszeit. Bei dem geschilderten Fall hat man einen noch höheren Druck und noch höhere Temperaturen als beim Autoklavieren - und das sollen diese Bakterien überleben! Das ist schwer $z u$ verstehen.

Gunkel: Die Erklärung lautet wohl, daß denaturiertes Eiweiß einen größeren Raum einnimmt als lebendes Eiweiß und es bei sehr hohen Drucken, nach dem Gesetz des geringsten Zwanges, nicht zur Denaturierung kommt.

WaLlHäUSSER: Ja, genau!

GuNkel: Noch eine Frage betreffend die Grenzflächen zwischen OOl und Wasser und bakterielle Aktivität. LA Rrvì̀re (1955a, b) zeigte, daß von den Bakterien selbst während des Abbaues solche Emulgatoren gebildet werden. 
WaLlHäUSSER: Auch das haben wir in Heizöltanks beobachtet. Aber es muß nicht immer der Fall sein. Wir haben bei unseren Laborversuchen die mit Sand beschickten Kulturflaschen zunächst mit Wasser aufgefüllt und die letzten $0.5 \mathrm{~cm}$ der Sandschicht mit OO1 überschichtet. Das Wachstum der Desulfovibrionen setzte ungefähr 0,5 bis $1,0 \mathrm{~cm}$ unter der Oberfläche ein und war erkennbar an der Schwarzfärbung. Hätten irgendwelche löslichen Substanzen eine Rolle gespielt, dann hätte diese Schwarzfärbung einen größeren Raum einnehmen müssen. Das war aber nicht der Fall. Wir können also schlußfolgern: Ol wird hier als Nährstoff benutzt. Wir wissen noch nicht, welche Fraktion des Ols die bedeutende Rolle spielt. Diese Untersuchungen werden wir an solchen Organismen durchführen, die tatsächlich einen stärkeren Abbau zeigen. Aber wir betreiben zunähst nur ein Screening-Programm, das uns zeigen soll, welche Organismen von Bedeutung sind. Bei solch geringen Abbaumengen sollte man sich damit nicht beschäftigen, denn das lohnt nicht. In dieser Hinsicht scheinen die Streptomyceten etwas interessanter zu sein.

\section{SснӧBERE: Verwandten Sie Reinkulturen?}

WALLHÄUSSER: Reinkulturen von Desulfovibrionen sind sehr schwer herzustellen. Sie sind meistens vergesellschaftet mit anderen Bakterien, und man muß mit sehr viel List und Tüicke herangehen, zum Beispiel mit Antibiotika, Penicillin oder Streptomycin, je nach dem, um welche grampositive oder gramnegative Verunreinigung es sich dabei handelt.

SсHÖвERL: Enthält das Erdöl nicht Beimischungen von Karbonsäuren und stickstoffhaltigen Verbindungen? Dann bestünde do.h die Möglichkeit, daß dieses Wachstum gar nicht auf Kosten der Kohlenwasserstoffe geht, sondern vielmehr auf schon oxydierten Kohlenstoffen beruht.

WALlHäusser: Das ist durchaus möglich; aber das läßt sich in diesem Versuch nicht ausschalten.

SCHÖBERL: Soweit ich informiert bin, konnte es bisher in keinem Fall bewiesen werden, daß Desulfovibrio in Reinkulturen überhaupt Kohlenwasserstoffe abbauen kann. Selbst ZoBeLL hat doch mit unreinen Kulturen gearbeitet.

WALLHÄUsSER: Ich habe mit Reinkulturen gearbeitet. Ein Wachstum der Kulturen ist festgestellt worden, aber welche Stoffe angegriffen werden, kann ich zunächst nicht sagen. Das wird erst durch eine saubere Analytik zu klären sein. Einer solchen, nich ganz einfachen Aufgabe unterzieht man sich erst dann, wenn man einen Effekt sieht, der diese Arbeit lohnend erscheinen läßt. Bei einem solch geringen Abbau lohnt es nicht, sich damit näher zu beschäftigen. Bei den Streptomyceten untersuchen wir 60 verschiedene Kulturen. Diese Arbeit wird im nächsten Jahr im Archiv für Mikrobiologie veröffentlicht werden. Von den Streptomyceten haben wir zunächst zwei Gruppen bearbeitet, die sogenannte blat-grün versporende und die grau versporende Gruppe.

SCHÖвERL: Haben Sie hierbei den aeroben Abbau untersucht?

WALLhÄUSSER: Ja, in Schüttelkulturen wohlgemerkt; die Laufzeit dieser Schüttelkulturen beträgt 14 Tage, wobei ein beträchtliches Wachstum auftritt.

SснӧвеRL: Mit welchen Flüssigkeitsmengen arbeiten Sie?

WatlhäUsSER: Mit $80 \mathrm{ml}$ in $300 \mathrm{ml}$ Erlenmeyerkolben. Die Schüttelfrequenz ist $200 \mathrm{Um}$ drehungen $/ \mathrm{min}$. bei einer Amplitude von $20 \mathrm{~cm}$ auf Rotationsschüttelapparaturen.

SCHWEISSFuRth: Wird der pH-Wert bei den Streptomycetenkulturen stark abgesenkt?

WaLlhäUSSER: Das ist recht unterschiedlich. Wir arbeiten mit Puffern, und zwar im optimalen Bereich 6,5. Wir haben Stämme, die pH-Werte bis 5 und andere, die pH-Werte bis 8 vertragen.

Gunkex: Haben Sie eine Vorstellung über die Herkunft des Schwefelwasserstoffes? Wieviel Prozent stammen von schwefelhaltigen, organischen Verbindungen des Ols und wieviel Prozent des gebildeten Schwefelwasserstoffes von Sulfaten?

WallhäUSSER: Ich habe für Ol keine genauen Zahlen. Diese sind sehr gering im Speichergas, weil vorher, bevor das Gas eingelagert wird, eine Schwefelwasserstoffwäsche durchgeführt wird. Ein Teil des im Speicher gebildeten Schwefelwasserstoffes wird als Pyrit festgelegt. Wir 
haben Pyrit von unterschiedlicher Größe zu Tage gefördert, und Herr Puchelt ist jetzt dabei, festzustellen, ob es rezenter ader fossiler Pyrit ist.

Gunkel: Meine Frage betraf die Herkunft des Sulfidschwefels. Ich möchte mich dagegen wehren, daß prinzipiell als Ursache jeder Schwefelwasserstoffbildung die Sulfatreduktion angesehen wird. In manchen marinen Sedimenten stammt $\mathrm{H}_{2} \mathrm{~S}$ za uber $50 \%$ aus organischen Schwefelquellen (GUNker \& Oppenhermer 1962).

WALlhäUSSER: Ich hatte schon betont, daß soviel organischer Restschwefel nach der $\mathrm{H}_{2} \mathrm{~S}$ Wäsche keinesfalls im Gas verbleibt. Der Schwefelgehalt des eingelagerten Gasgemisches ist nahezu konstant; er steigt erst in dem Speicher sehr rasch und ganz erheblich, und wir sind sicher, daß es sich hierbei um eine $\mathrm{H}_{2} \mathrm{~S}$-Produktion durch Desulfovibrionen handelt. Vorher wurde kein Pyrit in der Lagerstätte nachgewiesen.

Gunkel: Wir haben auch in marinen Sedimenten eine rezente Pyritbildung.

WallhäUSSER: Ja, die ist zum Teil sehr stark, auch in den Untertagespeichern. Wir fördern ab und $\mathrm{zu}$ einmal Sand zutage, der schwarz verklebt und zusammengebacken ist.

Gunkel: Auch Desulfovibrio kann Schwefel aus organischen Quellen als $\mathrm{H}_{2} \mathrm{~S}$ freisetzen.

Watlhäusser: Das ist noch nicht geklärt. Mit dieser Frage haben wir uns nicht befaßt, aber die Herren von der Ruhrgas sind der Meinung, daß die Mengen an organischen Schwefelverbindungen so gering sind, daß sie auf keinen Fall in die Rechnung miteingehen. Bei einer Reihe von Schwefelquellen ist im Grundwasser kein Sulfat vorhanden. Darüber, an der Oberfläche, befinden sich jedoch Markasitlagerstätten. In diesen produziert nun Thiobazillus thiooxydans aus dem Pyrit Sulfat und dieses wandert dann in das Grundwasser ein und dient dort als $\mathrm{SO}_{4-}$ Quelle für diese Desulfovibrionen.

SснӧвеRL: Sie bemerkten, Sie hätten im Untertagegasspeicher eine Symbiose von verschiedenen Arten gefunden. Haben Sie diese Arten einmal zusammengebracht?

Wallhäusser: Nein, wir haben diese Organismen erst vor etwa einem Monat isoliert, und wir wissen nicht, ob sie $\mathrm{O} l$ abbauen. In diesem Untertagegasspeicher, aus dem sie stammen, sind $z$ war Spuren von Ol vorhanden, aber auch Spuren von Erdgas.

SchöвERx: Man hat doch bei dem Kohlenwasserstoffabbau vielfach festgestellt, daß Reinkulturen nur geringe Aktivität haben, während sich Mischkulturen durch außerordentlich intensive Abbauleistungen auszeichnen.

WALlHäUSSER: Das müßte untersucht werden. Hier haben wir sicherlich einiges versäumt, und $z$ war bei all diesen Erdölfeldern, denn wir haben uns zunächst um andere Mikroorganismen nicht gekümmert, sondern wir haben uns nur mit den Desulfoxibrionen befaßst die Schwefelwasserstoff bilden. Was die anderen Mikroorganismen vermögen, wissen wir noch nicht.

BLOKKER: Ist überhaupt genügend Sulfat vorhanden?

Wallhäusser: Das ist Voraussetzung, sonst geht ein Abbau nicht vonstatten. Bei dem Speicher "Hähnlein" vermuten wir, daß dieses Sulfat manchmal nicht ausreicht. Es war ganz interessant festzustellen, daß die Entnahmesonden nicht gleiche Mengen an Schwefelwasserstoff liefern. Es gibt dort eine Reihe von Ersheinungen, für die wir noch keine rechte Exklärung haben. An manchen Entnahmesonden ist bei der Entnahme eine Temperaturerhöhung festzustellen; die Speichertemperatur liegt bei $25^{\circ} \mathrm{C}$, und die Entnahmetemperatur für einige Sonden steigt auf über $100^{\circ} \mathrm{C}$ an. Sind das autokatalytische Prozesse, die hier zu diesem Temperaturanstieg führen, oder sind es vielleicht die Bakterien, die dafür verantwortlich zu machen sind? Ich denke dabei an die Selbstentzündung von $\mathrm{Heu}$, wo es auch zu solch hohen Temperaturen kommen kann. Oder ist es ein rein demischer Prozeß? Wir wissen es noch nicht.

BLOKKeR: In dem eiweißreichen Milieu des Heues ist die Stoffwechselgeschwindigkeit doch wesentlich höher.

WALLH ÄUSSER: Wir fanden bei unseren Laborversuchen durchaus nicht diesen lebhaften Stoffwechsel, wie er in den Untertagegasspeichern oder aber auch in den Erdölfeldern zu erwarten ist. 
GUNKEL: Vielleicht würden Sie einen intensiveren Stoff wedhsel mit Mischkulturen erhalten. Sie haben ja aus einer komplexen Lebensgemeinsdaft nur eine Art herausgenommen, die Sie untersuchen.

WALLhäUSSER: Das ist durchaus möglich.

BLOKKER: Kann errechnet werden, wieviel Sulfat zur Verfügung steht?

WALLHäUSSER: Wir haben von dem Grundwasser Analysen gemacht und mußten feststellen, daß ausreichende Sulfatmengen vorhanden sind. Nur im Speicher "Hähnlein“ ist der Sulfatgehalt variabel. Vielleicht reguliert eine Wasserader den Sulfatzufluß.

BLoKKeR: Können Sie den Gasspeicher nicht impermeabel machen?

WALLhäuSSER: Nein, das ist sehr schwierig. Das geologische Geschehen in „Hähnlein“, im Rheingraben, ist noch zu übersehen, nicht jedoch oben bei Engelbostel, wo zahlreiche Schichten und Verwerfungen auftreten.

Blokker: Sie kennen vielleicht die Lage in Schombeck, wo man O1 mit Wasser aufpumpt. Das Wasser hat jedoch einen Salzgehalt von $10 \%$, so daß man es in Oberflächengewässern nicht loswerden kann; es muß daher wieder hinuntergepumpt werden.

WALLHÄUSSER: Das wird in fast allen Sekundärförderungsanlagen so gemacht.

Blok ker: $\mathrm{D}$ a wird die Trennung von $\mathrm{Ol}$ und Wasser belüftet, eine Flotation. Dann setzt sich noch ein Teil des Eisens als Ferrit ab. Man kann jedoch - um Verstopfungen zu vermeiden - trübes Wasser nicht wieder hinunterpumpen. Es wird so lange weiterbeliftet, bis alles Eisen in Ferrit überführt ist; dann wird es filtriert. Man bekommt somit klares Wasser ohne Schwebestoffe. Es kann jedoch wegen des hohen Sauerstoffgehalts noch nicht hinunter.

WALLHÄUSSER: In dieser Weise verfährt man auch bei dem Kreislauf des Injektionswassers. In Untertagegasspeichern beobachtet man gelegentlich noch eine andere Erscheinung. Beim Offnen der Auslaßsonden tritt mitunter eine ziemlich starke Abgabe von $\mathrm{H}_{2} \mathrm{~S}$ auf, während bei anderen Sonden der $\mathrm{H}_{2} \mathrm{~S}$-Anteil wesentlich geringer ist. Wie kommt es nun zu diesem Unterschied in einem Speicher und bei Sonden, die vielleicht nur einen Abstand von $200 \mathrm{~m}$ haben? Wir denken an folgende Erklärung: Ein. Teil des $\mathrm{H}_{2} \mathrm{~S}$ löst sich unter Druck im Wasser und in dem Augenblick, in dem es zu einer Entspannung kommt, werden erhöhte Mengen an $\mathrm{H}_{2} \mathrm{~S}$ frei.

BLokkeR: Ich möchte hoffen, daß Ihre mikrobiologischen Prozesse auch auftreten, wenn Grundwasser mit $O \mathrm{Ol}$ verunreinigt ist!

SCHÖBERL: Ich habe noch eine methodische Frage. Um welche Verschlüsse handelt es sich bei den verwendeten Kulturgefäßen?

WALlhäusser: Es sind Gummistopfen, die durch eine Metallkappe festgehalten werden. Wir geben dazu kleine Kunststoffspritzen, und der Techniker nimmt damit eine Probe aus dem zu untersuchenden Wasser, spritzt nun einen Kubikzentimeter in die Testflasche und beobachtet diese über 14 Tage (bei Zimmertemperatur). In der Regel muß eine Schwarzfärbung nach ungefähr 14 Tagen bei normalen Bedingungen auftreten. Sie kann länger dauern, wenn der Stamm eine andere Kochsalztoleranz hat; dann kann es mitunter vorkommen, daß erst nach 8 Wochen eine Schwarzfärbung erfolgt. Deshalb ist es sehr wichtig, daß man entsprechende Flaschen nimmt. Wir haben solche mit $0 \%, 3 \%, 5 \%, 7,5 \%, 10 \%$ und $15 \%$ Kochsalzgehalt. Der Speicher in "Hähnlein" hat $6 \%$ Kochsalz, während in Eschenfelden, in der gleichen Tiefe etwa $550 \mathrm{~m}$ bis $580 \mathrm{~m}$ bei einer Speichermächtigkeit von etwa $40 \mathrm{~m}$ - Süßwasser vorliegt. Dort können wir einen derartigen Desulfovibrio-Stamm schlecht identifizieren. Wir wissen nicht, ob er oberflächlich mit der Bohrspülung eingeschleppt wurde oder über Verwerfungen einwanderte. In „Hähnlein" wurden einige Bohrungen „aseptisch" durchgeführt. In der letzten Phase, kurz vor der Berührung mit dem Speicherhorizont, wurde das mit 1000 p.p.m. Desinfektionsmittel versetzte Bohrspülwasser herausgenommen und durch chloriertes Trinkwasser ersetzt. Aus dem Speicherbereich wurde mit Hilfe eines Gummikernrohres ein etwa $20 \mathrm{~m}$ langer Kern gezogen. Diesen Gummischlauch haben wir an Ort und Stelle zerlegt; er ist etwa $8 \mathrm{~cm}$ dick, und in yorbereitete Konservendosen wurden einige Scheiben aus diesem Kern herausgeschnitten. 
Diese aus den verschiedenen Tiefen stammenden Proben wurden dann auf ihren Keimgehalt untersucht. Dieser Speicher wird von grauen Tonlinsen durchsetzt und die Tonlinsen haben natürlich auch für die Strömungsverhältnisse im Speicher eine gewisse Bedeutung.

\section{Mikroorganismen als Ursache der Korrosion in Lager- und Treibstofftanks}

GUNKEL: Sie erwähnten in Threm Vortrag durch Bakterien hervorgerufene Korrosionen in Lagertanks. Diese können auch in Zweitaktmotoren auftreten, die längere Zeit nicht benutzt wurden. Es kann sich Kondenswasser bilden, und das OI liefert die C-Quelle.

WALLḦ̈USSER: Wir haben Mikroorganismen in größeren Mengen aus den Tanks verschiedener Flugzeuge isolieren können.

BLокKеR: Es genügt ja ein ganz dünner Wasserfilm an den Wandungen der Tanks.

WallhäUsser: Hinzu kommt das Schwitzwasser, denn diese Tanks sind ja belüftet. Wird feuchte Luft aufgenommen, so scheidet sich dann in großer Höhe und bei niedriger Außentemperatur das Wasser ab. Das ganze Geschehen spielt sich stets an der Grenzfläche zwischen $\mathrm{O} 1$ und Wasser ab. Diese ist besonders bei Emulsionen sehr groß; deshalb geht dieser Stoffumsatz hier viel schneller vor sich, aud der Abbau des Emulgators. Bei vielen Schneidölen wird nicht Ol, sondern der Emulgator abgebaut, es kommt nacher zu einer Entmischung, und der Olfilm schwimmt oben darauf. Der Lochfraß in diesen Tanks ist manchmal ganz beachtlich. Es werden in kürzester Zeit schon einige millimetertiefe Löcher herausgefressen, besonders bei Maschinen, die lange stehen. Dies triff auch für unsere Haushaltstanks (Olheizung) zu. Hier kann man sich helfen, indem man an der einen Seite des schräg gelagerten Tanks ein Entnahmehähnchen anbringt, und dann ab und zu das Kondenswasser abläßst. So kann man diese Gefahren am besten beheben. Wir haben natürlich auch die Möglichkeit, durch Zusatz von Konservierungsmitteln das Wachstum der Bakterien zu unterdrücken. Diese Methode stößt gerade bei der Luftfahrtindustrie auf stärkste Bedenken. Es müssen dort erst Untersuchungen über das Verhalten dieser Desinfektionszusätze auf die Gesamtverbrennung durchgeführt werden. Bisher hilft man sich durch häufiges Ablassen des Kondenswassers.

SCHWEISFURTH: Dies dürfte bei den häuslichen Heizöltanks wohl nicht so leicht möglich sein.

WALLHÄUSSER: Ja, das geht; bei den heurigen, modernen Tanks ist ein kleiner Ablaßhahn eingebaut. Damit kann man das Wasser herauslassen. Man beklagt mit Recht die Verschmutzung des Meerwassers durch $O 1$, aber es wird mehr Wasser auf dem Lande verschmutzt werden, wenn in einigen Jahren Heizöltanks durchrosten. Dieses Öl wird sicherlich in unser Trinkwasser eindringen. Dort haben wir keine Handhabe! Heute wird ein vorbeugender Schutz gefordert, indem der Tank in eine Olwanne hineingestellt werden muß, aber um all die früher vergrabenen Tanks kümmert sich zunächst niemand.

KüHL: Die Mischung Wasser-Ol kann auch bei Tankern zu bedeutenden Korrosionserscheinungen führen. Vor einigen Jahren wurde in Hamburg zur Lösung dieses Problems eine internationale Tagung durchgeführt. Korrosionen in den Tankern traten dadurch auf, daß bei diesen Schiffen einige Tanks abwechselnd mit OI und Ballastwasser gefahren wurden. Das Problem wurde dadurch gelöst, daß nur bestimmte Tanks für den Wedsel OI-Wasser benutzt wurden. Außerdem wurde bei der Konstruktion darauf geachtet, schlecht zu reinigende Ecken und Winkel zu vermeiden.

WALLHÄUSSER: Wir haben auch Tanker untersudt und fanden ebenfalls sehr hohe Keimzahlen. Man kann diese Infektionskette verfolgen, und zwar yon den Tankern zunächst zu den kleinen Flußtankschiffen und dann zu dem Großtank bei der Raffinerie, obwohl ja dort eine Unterbrechung der Infektionskette erfolgen sollte. Wir konnten feststellen, daß hier auch die Cladosporien, die aus den Tanks der Düsenflugzeuge isoliert wurden, bei den abgebenden Olfirmen vorhanden sind. Sie kommen meist nicht durch die Luft, sondern auf dem Transportwege. Das gilt auch für die Bohrspülungen. Hierzu werden häufig Transportfahrzeuge genommen, die 